\title{
On a Class of Hermite Interpolation Polynomials for Nonlinear Second Order Partial Differential Operators
}

\author{
Leonid A. Yanovich ${ }^{1, \star}$ and Marina V. Ignatenko ${ }^{2, \star \star}$ \\ ${ }^{1}$ Institute of Mathematics, National Academy of Sciences of Belarus, Surganova Str. 11, 220072 Minsk, \\ Belarus \\ ${ }^{2}$ Faculty of Mechanics and Mathematics, Belarus State University, Nezavisimosti Ave. 4, 220030 Minsk, \\ Belarus
}

\begin{abstract}
This article is devoted to the problem of construction of Hermite interpolation formulas with knots of the second multiplicity for second order partial differential operators given in the space of continuously differentiable functions of two variables. The obtained formulas contain the Gateaux differentials of a given operator. The construction of operator interpolation formulas is based on interpolation polynomials for scalar functions with respect to an arbitrary Chebyshev system of functions. An explicit representation of the interpolation error has been obtained.
\end{abstract}

\section{Introduction}

The interpolation of the functions provides the basis for the construction and research of approximate and numerical methods for the solution of many classes of problems. In particular, it is widely used for the approximate representation and calculation of functions, the numerical integration and differentiation, the construction of approximate methods for solving various classes of linear and nonlinear equations, etc. It is natural and necessary an extension of the interpolation theory of the functions to other mathematical objects. The operator interpolation, as one of the sections of applied functional analysis and the general theory of approximate methods, significantly generalizes the problem of function interpolation and is the foundation for the construction of approximate methods and algorithms to solve applied problems. The main problems of the operator interpolation are formulated similarly to the classical problems of the function interpolation.

A number of other interpolation formulas for operators given in the spaces of functions and matrices are given in [1]-[4].

\section{Interpolation operator formulas of the Hermite type}

Let $X=X(T \times S)$ be a given space of smooth functions on $T \times S \subseteq R^{2}$. By $\delta F[x ; h]$ we denote the Gateaux differential at the point $x=x(t, s)$ in the direction $h=h(t, s)(x, h \in X)$ for an operator $F(x)$

${ }^{\star}$ e-mail: yanovich@im.bas-net.by

$\star \star$ e-mail: ignatenkomv@bsu.by 
defined on the set $X$. We consider the operator polynomials of the form

$$
P_{2 n+1}(x)=\sum_{k=0}^{2 n+1} a_{k}(\xi, t, s) \varphi_{k}(x(t, s)),
$$

where $a_{k}(\xi, t, s)$ are arbitrarily given functions of the variables $\xi, t$ and $s$, and $\left\{\varphi_{k}(x)\right\}_{k=0}^{2 n+1}$ is some arbitrary Chebyshev system of functions.

We consider the Hermite interpolation operator formulas with respect to knots of the second multiplicity. In [5] we have constructed the operator polynomial of the Hermite type containing the Gateaux differentials of a given operator of the form

$$
H_{2 n+1}(F ; x)=F\left(x_{0}\right)+\sum_{k=1}^{n} \int_{0}^{1} \delta F\left[x_{0}+\tau\left(x_{k}-x_{0}\right) ; \frac{h_{n, k}(x)}{\sigma_{n}(x)}\left(x_{k}-x_{0}\right)\right] d \tau+\sum_{k=0}^{n} \delta F\left[x_{k} ; \frac{q_{n, k}(x)}{\sigma_{n}(x)}\right],
$$

where $h_{n, k}(x)$ and $q_{n, k}(x)$ are the Hermite fundamental polynomials of the $(2 n+1)$-degree of the form (1) in the case of second multiplicity knots $x_{0}, x_{1}, \ldots, x_{n}$ with respect to some Chebyshev system $\left\{\varphi_{k}(x)\right\}_{k=0}^{2 n+1}$ of functions, for which $h_{n, k}\left(x_{j}\right)=q_{n, k}^{\prime}\left(x_{j}\right)=\delta_{k j}, h_{n, k}^{\prime}\left(x_{j}\right)=q_{n, k}\left(x_{j}\right)=0(k, j=0,1, \ldots, n)$, and $\sigma_{n}(x)=\sum_{k=0}^{n} h_{n, k}(x)$ is a constant or a variable value. For example, if $\left\{\phi_{k}(x)=x^{k}\right\}_{k=0}^{2 n+1}$ is an algebraic system of functions, then the fundamental polynomials $h_{n, k}(x)$ and $q_{n, k}(x)$ are defined by the rules

$$
h_{n, k}(x)=l_{n, k}^{2}(x)\left[1-\frac{\omega_{n}^{\prime \prime}\left(x_{k}\right)}{\omega_{n}^{\prime}\left(x_{k}\right)}\left(x-x_{k}\right)\right], q_{n, k}(x)=l_{n, k}^{2}(x)\left(x-x_{k}\right), k=0,1, \ldots, n,
$$

where $l_{n, k}(x)=\prod_{j=0 ; j \neq k}^{n} \frac{x-x_{j}}{x_{k}-x_{j}}$ are algebraic Lagrange fundamental polynomials. In this case $\sigma_{n}(x) \equiv$ 1 , and from the interpolation nodes it is required that $x_{k}(t, s)-x_{j}(t, s) \neq 0(k \neq j)$ for all $(t, s) \in T \times S$.

The polynomial (2) is the interpolation operator for a given operator $F(x)$ on the set $X$ relative to the knots $x_{0}, x_{1}, \ldots, x_{n}$ of the second multiplicity. It satisfies the following conditions:

$$
H_{2 n+1}\left(F ; x_{k}\right)=F\left(x_{k}\right), \delta H_{2 n+1}\left[x_{k} ; h\right]=\delta F\left[x_{k} ; h\right](k=0,1, \ldots, n ; h \in X) .
$$

For the interpolation error $r_{2 n+1}(x)=F(x)-H_{2 n+1}(F ; x)$, where $H_{2 n+1}(F ; x)$ is the interpolation polynomial (2), the following representation holds:

$$
r_{2 n+1}(x)=\sum_{k=1}^{n+1} \int_{0}^{1} \delta F\left[x_{0}+\tau\left(x_{k}-x_{0}\right) ;\left(\frac{h_{n+1, k}(x)}{\sigma_{n+1}(x)}-\frac{h_{n, k}(x)}{\sigma_{n}(x)}\right)\left(x_{k}-x_{0}\right)\right] d \tau+\sum_{k=0}^{n+1} \delta F\left[x_{k} ; \frac{q_{n+1, k}(x)}{\sigma_{n+1}(x)}-\frac{q_{n, k}(x)}{\sigma_{n}(x)}\right],
$$

where $x_{n+1}=x, h_{n, n+1}(x)=q_{n, n+1}(x) \equiv 0$.

\section{Interpolation formulas of the Hermite type for second order partial differential operators}

There exists a rich literature on the theory of partial differential equations and operators. See, for example, [6]-[9] and others. We consider the second order partial differential operators $F: C^{2}(T \times$ $S) \rightarrow Y$ of the form

$$
F(x)=f\left(t, s, x(t, s), x_{t}^{\prime}(t, s), x_{s}^{\prime}(t, s), x_{t^{2}}^{\prime \prime}(t, s), x_{t, s}^{\prime \prime}(t, s), x_{s^{2}}^{\prime \prime}(t, s)\right),
$$

where $x_{t}^{\prime}(t, s)=\frac{\partial x(t, s)}{\partial t}, x_{t, s}^{\prime \prime}(t)=\frac{\partial^{2} x(t, s)}{\partial t \partial s}, x_{t^{2}}^{\prime \prime}(t, s)=\frac{\partial^{2} x(t, s)}{\partial t^{2}}$, the derivative $x_{t, s}^{\prime \prime}(t, s)=x_{s, t}^{\prime \prime}(t, s), C^{2}(T \times S)$ is the space of two times continuously differentiable functions $x(t, s)$ on $T \times S \subseteq R^{2}$, the function 
$y=f\left(t, s, u_{0}, u_{1}, \ldots, u_{5}\right)$ is defined on the rectangle $\Omega=T \times S \times T_{0} \times T_{1} \times \cdots \times T_{5}, T_{i}$ are sets of the number line $(i=0,1, \ldots, 5)$, and $Y$ is a function space.

We construct the interpolation polynomial for the operator (5) using the equality (2). The differential operator (5) depends on the one functional variable $x(t, s)$, for it the Gateaux differential $\delta F[x ; h]$ at the point $x=x(t, s)$ in the direction $h=h(t, s)\left(x, h \in C^{2}(T \times S)\right)$ is calculated according to the rule

$$
\begin{gathered}
\delta F[x ; h]=\frac{\partial f}{\partial x} h(t, s)+\frac{\partial f}{\partial x_{t}^{\prime}} h_{t}^{\prime}(t, s)+\frac{\partial f}{\partial x_{s}^{\prime}} h_{s}^{\prime}(t, s)+\frac{\partial f}{\partial x_{t^{2}}^{\prime \prime}} h_{t^{2}}^{\prime \prime}(t, s)+\frac{\partial f}{\partial x_{t, s}^{\prime \prime}} h_{t, s}^{\prime \prime}(t, s)+\frac{\partial f}{\partial x_{s^{2}}^{\prime \prime}} h_{s^{2}}^{\prime \prime}(t, s)= \\
=\sum_{i, j=0 ; i+j \leq 2}^{2} \frac{\partial f\left(t, s, x(t, s), x_{t}^{\prime}(t, s), x_{s}^{\prime}(t, s), x_{t^{2}}^{\prime \prime}(t, s), x_{t, s}^{\prime \prime}(t, s), x_{s^{2}}^{\prime \prime}(t, s)\right)}{\partial x_{t^{i}, s^{j}}^{(i+j)}} h_{t^{i}, s^{j}}^{(i+j)}(t, s),
\end{gathered}
$$

where $x_{t^{i}, s^{j}}^{(i+j)}(t, s)=\frac{\partial^{i+j} x(t, s)}{\partial t^{i} \partial s^{j}}(i, j=0,1,2 ; i+j \leq 2)$. Bearing in mind the rule (6), the formula (2) for the operators (5) takes the form

$$
\begin{gathered}
H_{2 n+1}(F ; x)=F\left(x_{0}\right)+\sum_{k=0}^{n} \sum_{i, j=0 ; i+j \leq 2}^{2} \frac{\partial F\left(x_{k}(t, s)\right)}{\partial\left(\frac{\partial^{i+j} x_{k}}{\partial t^{i} \partial s^{j}}\right)} \frac{\partial^{i+j}}{\partial t^{i} \partial s^{j}} \frac{q_{n, k}(x(t, s))}{\sigma_{n}(x(t, s))}+ \\
+\sum_{k=1}^{n} \int_{0}^{1} \sum_{i, j=0 ; i+j \leq 2}^{2} \frac{\partial}{\partial\left(\frac{\left.\partial^{i+j} v_{k}\right)}{\partial t^{i} \partial s^{j}}\right)} f\left(t, s, v_{k}(t, s, \tau), \frac{\partial v_{k}(t, s, \tau)}{\partial t}, \frac{\partial v_{k}(t, s, \tau)}{\partial s} \frac{\partial^{2} v_{k}(t, s, \tau)}{\partial t^{2}},\right. \\
\left.\frac{\partial^{2} v_{k}(t, s, \tau)}{\partial t \partial s}, \frac{\partial^{2} v_{k}(t, s, \tau)}{\partial s^{2}}\right) \frac{\partial^{i+j}}{\partial t^{i} \partial s^{j}}\left\{\frac{h_{n, k}(x(t, s))}{\sigma_{n}(x(t, s))}\left(x_{k}(t, s)-x_{0}(t, s)\right)\right\} d \tau
\end{gathered}
$$

where $v_{k}=v_{k}(t, s, \tau)=x_{0}(t, s)+\tau\left(x_{k}(t, s)-x_{0}(t, s)\right), k=1,2, \ldots, n$.

The polynomial (7) satisfies the interpolation conditions (3), where $X=C^{2}(T \times S)$.

We may obtain the representation of the interpolation error of the operator $F(x)$ by the polynomial (7) and taking into account the equality (4). We have

$$
\begin{gathered}
r_{2 n+1}(x)=\sum_{k=0}^{n+1} \sum_{i, j=0 ; i+j \leq 2}^{2} \frac{\partial F\left(x_{k}(t, s)\right)}{\partial\left(\frac{\partial^{i+j} x_{k}}{\partial t^{i} \partial s^{j}}\right)} \frac{\partial^{i+j}}{\partial t^{i} \partial s^{j}}\left(\frac{q_{n+1, k}(x(t, s))}{\sigma_{n+1}(x(t, s))}-\frac{q_{n, k}(x(t, s))}{\sigma_{n}(x(t, s))}\right)+ \\
+\sum_{k=1}^{n+1} \int_{0}^{1} \sum_{i, j=0 ; i+j \leq 2}^{2} \frac{\partial}{\partial\left(\frac{\partial^{i+j} v_{k}}{\partial t^{i} \partial s^{j}}\right)} f\left(t, s, v_{k}(t, s, \tau), \frac{\partial v_{k}(t, s, \tau)}{\partial t}, \frac{\partial v_{k}(t, s, \tau)}{\partial s}, \frac{\partial^{2} v_{k}(t, s, \tau)}{\partial t^{2}},\right. \\
\left.\frac{\partial^{2} v_{k}(t, s, \tau)}{\partial t \partial s}, \frac{\partial^{2} v_{k}(t, s, \tau)}{\partial s^{2}}\right) \frac{\partial^{i+j}}{\partial t^{i} \partial s^{j}}\left\{\left(\frac{h_{n+1, k}(x(t, s))}{\sigma_{n+1}(x(t, s))}-\frac{h_{n, k}(x(t, s))}{\sigma_{n}(x(t, s))}\right)\left(x_{k}(t, s)-x_{0}(t, s)\right)\right\} d \tau,
\end{gathered}
$$

where $x_{n+1}=x, h_{n, n+1}(x)=q_{n, n+1}(x) \equiv 0$.

Example. Let us consider the second order partial differential operator

$$
F(x)=\phi(t, s)+a(t, s) x(t, s)+b(t, s) x^{p}(t, s)+c(t, s) x_{t}^{\prime}(t, s)+h(t, s) x_{s^{2}}^{\prime \prime}(t, s),
$$

where $p$ is a fixed non-negative integer number, and $\phi(t, s), a(t, s), b(t, s), c(t, s), h(t, s)$ are arbitrarily given functions of the variables $t$ and $s$. We construct the Hermite interpolation polynomial $H_{3}(F ; x)$ of the form (7) for the operator (8). As fundamental interpolation polynomials $h_{1, k}(x), q_{1, k}(x)$ $(k=0,1)$ we choose the algebraic polynomials

$$
h_{1, k}(x)=l_{1, k}^{2}(x)\left(1+2 l_{1,1-k}(x)\right), q_{1, k}(x)=l_{1, k}^{2}(x)\left(x-x_{k}\right),
$$

where $l_{1, k}(x)=\left(x-x_{1-k}\right) /\left(x_{k}-x_{1-k}\right), k=0,1$, and we take as interpolation nodes $x_{k}(t, s), k=0,1$, the system of functions $x_{0}(t, s) \equiv 3, x_{1}(t, s)=\sin t$. In this case the function $v_{1}(t, s, \tau)=3+\tau(\sin t-3)$, $\sigma_{1}(x) \equiv 1$. 
Bearing in mind that $\int_{0}^{1} p v_{1}^{p-1}(t, s, \tau)(\sin t-3) d \tau=\sin ^{p} t-3^{p}$ the Hermite interpolation formula (7) in the case $n=1$ for the operators (8) takes the form

$$
\begin{gathered}
H_{3}(F ; x)=\phi(t, s)+3 a(t, s)+3^{p} b(t, s)+\left(a(t, s)+p 3^{p-1} b(t, s)\right) q_{1,0}(x(t, s))+ \\
+\left(a(t, s)+p b(t, s) \sin ^{p-1} t\right) q_{1,1}(x(t, s))+\sum_{k=0}^{1}\left\{c(t, s) \frac{\partial}{\partial t} q_{1, k}(x(t, s))+h(t, s) \frac{\partial^{2}}{\partial s^{2}} q_{1, k}(x(t, s))\right\}+ \\
+a(t, s)(\sin t-3) h_{1,1}(x(t, s))+b(t, s)\left(\sin ^{p} t-3^{p}\right) h_{1,1}(x(t, s))+ \\
+c(t, s) \frac{\partial}{\partial t}\left\{h_{1,1}(x(t, s))(\sin t-3)\right\}+h(t, s) \frac{\partial^{2}}{\partial s^{2}}\left\{h_{1,1}(x(t, s))(\sin t-3)\right\}
\end{gathered}
$$

By direct calculations, we verify the validity of the interpolation conditions

$$
H_{3}^{(j)}\left(F ; x_{i}\right)=F^{(j)}\left(x_{i}\right)(i, j=0,1) .
$$

Really, in view of the equalities $h_{1,1}(3)=0, q_{1,0}(3)=0$ and $q_{1,1}(3)=0$ we obtain $H_{3}(F ; 3)=\phi(t, s)+$ $3 a(t, s)+3^{p} b(t, s)=F(3)$. As $q_{1,0}(\sin t)=q_{1,1}(\sin t)=0$, and $h_{1,1}(\sin t)=1$, then $H_{3}(F ; \sin t)=$ $\phi(t, s)+3 a(t, s)+3^{p} b(t, s)+b(t, s)\left(\sin ^{p} t-3^{p}\right)+a(t, s)(\sin t-3)+c(t, s) \cos t=\phi(t, s)+a(t, s) \sin t+$ $b(t, s) \sin ^{p} t+c(t, s) \cos t=F(\sin t)$.

Further, since $q_{1,0}^{\prime}(3)=q_{1,1}^{\prime}(\sin t)=1, q_{1,1}^{\prime}(3)=h_{1,1}^{\prime}(3)=0, q_{1,0}^{\prime}(\sin t)=h_{1,1}^{\prime}(\sin t)=0$, then $H_{3}^{\prime}(F ; 3)=a(t, s)+p 3^{p-1} b(t, s)=F^{\prime}(3), H_{3}^{\prime}(F ; \sin t)=a(t, s)+p b(t, s) \sin ^{p-1} t=F^{\prime}(\sin t)$. Thus, the Hermite interpolation formula (9) satisfies the condition (10).

\section{Conclusion}

The obtained results may be used in theoretical research as a basis for constructing approximate methods of solving some nonlinear operator equations that occur in nonlinear dynamics and mathematical physics.

\section{References}

[1] L. A. Yanovich and M. V. Ignatenko, Mathematical Models and Computer Simulations 26, 11, 90-96 (2014)

[2] L. A. Yanovich and M. V. Ignatenko, Proc. of NAS of Belarus, Series of Physical-Mathematical Sciences 2, 7-23 (2017) (in Russian)

[3] V. L. Makarov, V. V. Khlobystov, and L. A. Yanovich, Methods of Operator Interpolation (Institute of Mathematics of NAS of Ukraine, Kiev, 2010) $517 \mathrm{pp}$.

[4] L. A. Yanovich and M. V. Ignatenko, Basics of the Interpolation Theory of Functions of Matrix Variables (in Russian, Belaruskaya Navuka, Minsk, 2016) 281 pp.

[5] M. V. Ignatenko and L. A. Yanovich, Proc. of NAS of Belarus, Series of Physical-Mathematical Sciences 1, 5-11 (2004) (in Russian)

[6] L. Hormander, Acta Mathematica 94, 161-248 (1955)

[7] R. Courant, Partial Differential Equations (Vol. II of Methods of Mathematical Physics by R. Courant and D. Hilbert, Interscience Publishers, New York, 1962) 811 pp.

[8] O. A. Ladyzhenskaya, The Boundary Value Problems of Mathematical Physics (Applied Mathematical Sciences 49, Berlin-Heidelberg-New York: Springer Verlag, 1985) 322 pp.

[9] S. Mizohata, The Theory of Partial Differential Equations (Cambridge University Press, 1973) 504 pp. 